\title{
THE FEYNMAN-SCHWINGER (WORLD-LINE) REPRESENTATION IN PERTURBATIVE QCD
}

\author{
Yu.A. Simonov ${ }^{1}$ and J.A. Tjon ${ }^{2}$ \\ ${ }^{1}$ Jefferson Laboratory, Newport News, USA \\ and
}

Institute of Theoretical and Experimental Physics, Moscow, Russia

${ }^{2}$ Institute for Theoretical Physics, University of Utrecht, and

KVI, University of Groningen, The Netherlands

The proper time path integral representation is derived explicitly for an arbitrary $n$ point amplitude in QCD. In the standard perturbation theory the formalism allows to sum up the leading subseries, e.g. yielding double-logarithm Sudakov asymptotics for form factors. Correspondence with the standard perturbation theory is established and connection to the Bern-Kosower-Strassler method is illustrated. 


\section{Dedication.}

During our studies in field theory we many times returned to the set of methods and ideas, which was developed by Misha Marinov. Here also belongs the path integral method in its different forms, with spin included in the quantummechanical world-line version, which is described below. The excellent review written by Misha on the subject which appeared in Physics Reports 1 was a most-read source on the subject at that time. It is a great pleasure and honour for us to dedicate the paper to his memory.

\section{Introduction.}

The present stage of development of field theory in general and of QCD in particular requires the exploiting of nonperturbative methods in addition to summing up perturbative series. This calls for specific methods where dependence on vacuum fields can be made simple and explicit. A good example is provided by the so-called Fock-Feynman-Schwinger Representation (FSR) based on the Fock-Schwinger proper time and Feynman path integral formalisme For QED asymptotic estimates the FSR was exploited in Ref. 4. Later on this formalism was rederived in Ref. 5 for scalar quarks in QCD and used in the framework of the stochastic background method in 6

More recently some modification of the FSR was suggested in Refs. 7,8. The one-loop perturbative amplitudes are especially convenient for FSR. These amplitudes were extensively studied in Refs. 9-11. Meanwhile the first extension of FSR to nonzero temperature field theory was done in 1213 This formed the basis of a systematic study of the role of nonperturbative (NP) configurations in the temperature phase transitions 12.14

One of the most important advantages of the FSR is that it allows to reduce physical amplitudes to weighted integrals of averaged Wilson loops. Thus the fields (both perturbative and NP) enter only throughWilson loops. For the latter case one can apply the cluster expansion method 15 which allows to sum up a series of approximations directly in the exponent. As a result one can avoid the summation of Feynman diagrams to get the asymptotics of form factors 16 The role of FSR in the treatment of NP effects is more crucial. In this case one can develop a powerful methof of background perturbation theor 17 treating the NP fields as a background 18

In the present paper the main focus will be on perturbative QCD, with the aim of establishing the correspondence between the standard perturbative expansion and FSR based expansion, stressing the point that FSR allows to make exponentiation in a very simple way. Finally also the relation of FSR to the popular Bern-Kosower-Strassler method is discussed. 


\section{General form of FSR in QCD.}

Let us consider a scalar particle (e.g. Higgs boson) interacting with the nonabelian vector potential, where the Euclidean Lagrangian is given by

$$
L_{\varphi}=\frac{1}{2}\left|D_{\mu} \varphi\right|^{2}+\frac{1}{2} m^{2}|\varphi|^{2} \equiv \frac{1}{2}\left|\left(\partial_{\mu}-i g A_{\mu}\right) \varphi\right|^{2}+\frac{1}{2} m^{2}|\varphi|^{2},
$$

Using the Fock-Schwinger proper time representation the two-point Green's function of $\varphi$ can be written in the quenched approximation as

$$
G(x, y)=\left(m^{2}-D_{\mu}^{2}\right)_{x y}^{-1}=\left\langle x\left|P \int_{0}^{\infty} d s e^{-s\left(m^{2}-D_{\mu}^{2}\right)}\right| y\right\rangle .
$$

To obtain the FSR for $G$ a second step is needed. As in Ref. 2 the matrix element in Eq. (2) can be rewritten in the form of a path integral, treating $s$ as the ordering parameter. Note the difference of the integral (2) from the case of the Abelian QED treated in Refs. 2,4,9: $A_{\mu}$ in our case is the matrix operator $A_{\mu}(x)=A_{\mu}^{a}(x) T^{a}$. It does not commute for different $x$. Hence the ordering operator $P$ in Eq. (2). The precise meaning of $P$ becomes more clear in the final form of a path integral

$$
G(x, y)=\int_{0}^{\infty} d s(D z)_{x y} e^{-K} P \exp \left(i g \int_{y}^{x} A_{\mu}(z) d z_{\mu}\right),
$$

where $K=m^{2} s+\frac{1}{4} \int_{0}^{s} d \tau\left(\frac{d z_{\mu}}{d \tau}\right)^{2}$. In Eq. (3) the functional integral can be written as

$$
(D z)_{x y} \simeq \lim _{N \rightarrow \infty} \prod_{n=1}^{N} \int \frac{d^{4} z(n)}{(4 \pi \varepsilon)^{2}} \int \frac{d^{4} p}{(2 \pi)^{4}} e^{i p\left(\sum_{n=1}^{N} z(n)-(x-y)\right)}
$$

with $N \varepsilon=s$. The last integral in Eq. (邦 ensures that the path $z_{\mu}(\tau), 0 \leq$ $\tau \leq s$, starts at $z_{\mu}(0)=y_{\mu}$ and ends at $z_{\mu}(s)=x_{\mu}$. The form of Eq. (3) is the same as in the case of QED except for the ordering operator $P$ which provides a precise meaning to the integral of the noncommuting matrices $A_{\mu_{1}}\left(z_{1}\right), A_{\mu_{2}}\left(z_{2}\right)$ etc. In the case of QCD the forms (3) and (4) were introduced in Refs. 5,6.

The FSR, corresponding to a description in terms of particle dynamics is equivalent to field theory, when all the vacuum polarisation contributions are also included $\mathrm{E}$ i.e.

$$
\begin{aligned}
& \sum_{N=0}^{\infty} \frac{1}{N !} \prod_{i=1}^{N} \int \frac{d s_{i}}{s_{i}} \int\left(D z_{i}\right)_{x x} \exp (-K) P \exp \left(i g \int_{y}^{x} A_{\mu}(z) d z_{\mu}\right) \\
& =\int D \varphi \exp \left(-\int d^{4} x L_{\varphi}(x)\right) .
\end{aligned}
$$


Both sides are equal to vacuum-vacuum transition amplitude in the presence of the external nonabelian vector field and hence to each other. For practical calculations proper regularization of the above equation has to be done.

The field $A_{\mu}$ in Eq. (1) can be considered as a classical external field or as a quantum one. In the latter case the Green's functions $\langle A . . A\rangle$ induce nonlocal current-current interaction terms in the l.h.s. of Eq. (5). Such terms can also be generated by the presence of a $\varphi$-field potential, $V(|\varphi|)$ in the r.h.s. of Eq. (5).

The advantage of FSR in this case follows from the very clear space-time picture of the corresponding dynamics in terms of particle trajectories. This is especially important if the currents can be treated as classical or static (for example, in the heavy quark case). The mentioned remark on usefulness of the FSR (3) becomes clear when one considers the physical amplitude, e.g. the Green's function of the white state $\operatorname{tr}\left(\varphi^{+}(x) \varphi(x)\right)$ or its nonlocal version $\operatorname{tr}\left[\varphi^{+}(x) \Phi(x, y) \varphi(y)\right]$, where $\Phi(x, y)$ - to be widely used in what follows - is the parallel transporter along some arbitrary contour $C(x, y)$

$$
\Phi(x, y)=P \exp \left(i g \int_{y}^{x} A_{\mu}(z) d z_{\mu}\right) .
$$

One has by standard rules

$$
\begin{aligned}
& G_{\varphi}(x, y)=\left\langle\operatorname{tr}\left[\varphi^{+}(x) \varphi(x)\right] \operatorname{tr}\left[\varphi^{+}(y) \varphi(y)\right]\right\rangle_{A} \\
& =\int_{0}^{\infty} d s_{1} \int_{0}^{\infty} d s_{2}(D z)_{x y}\left(D z^{\prime}\right)_{x y} e^{-K-K^{\prime}}\langle W\rangle_{A}+\ldots
\end{aligned}
$$

where dots stand for the disconnected part, $\left\langle G_{\varphi}(x, x) G_{\varphi}(y, y)\right\rangle_{A}$. We have used the fact that the propagator for the charge-conjugated field $\varphi^{+}$is proportional to $\Phi^{\dagger}(x, y)=\Phi(y, x)$. Therefore the ordering $P$ must be inverted, $\Phi^{\dagger}(x, y)=$ $P \exp \left(i g \int_{x}^{y} A_{\mu}(z) d z_{\mu}\right)$. Thus all dependence on $A_{\mu}$ in $G_{\varphi}$ is reduced to the Wilson loop average

$$
\langle W\rangle_{A}=\left\langle\operatorname{tr} P_{C} \exp i g \int_{C} A_{\mu}(z) d z_{\mu}\right\rangle_{A} .
$$

Here $P_{C}$ is the ordering around the closed loop $C$ passing through the points $\mathrm{x}$ and $\mathrm{y}$, the loop being made of the paths $z_{\mu}(\tau) a, z_{\mu}^{\prime}\left(\tau^{\prime}\right)$ and to be integrated over.

The FSR can also be used to describe the quark and gluon propagation. Similar to the QED case, the fermion (quark) Green's function in the presence 
of an Euclidean external gluonic field can be written as

$$
\begin{aligned}
& G_{q}(x, y)=\langle\psi(x) \bar{\psi}(y)\rangle_{q}=\left\langle x\left|\left(m_{q}+\hat{D}\right)^{-1}\right| y\right\rangle \\
& =\left\langle x\left|\left(m_{q}-\hat{D}\right)\left(m_{q}^{2}-\hat{D}^{2}\right)^{-1}\right| y\right\rangle \\
& =\left(m_{q}-\hat{D}\right) \int_{0}^{\infty} d s(D z)_{x y} e^{-K} \Phi_{\sigma}(x, y),
\end{aligned}
$$

where $\Phi_{\sigma}$ is the same as was introduced in Ref. 2

$$
\Phi_{\sigma}(x, y)=P_{A} \exp \left(i g \int_{y}^{x} A_{\mu} d z_{\mu}\right) P_{F} \exp \left(g \int_{0}^{s} d \tau \sigma_{\mu \nu} F_{\mu \nu}\right)
$$

and $\sigma_{\mu \nu}=\frac{1}{4 i}\left(\gamma_{\mu} \gamma_{\nu}-\gamma_{\nu} \gamma_{\mu}\right)$, while $K$ and $(D z)_{x y}$ are defined in Eqs. (3) and (画. Note that operators $P_{A}, P_{F}$ in Eq. (10) preserve the proper ordering of matrices $A_{\mu}$ and $\sigma_{\mu \nu} F_{\mu \nu}$ respectively. Explicit examples are considered below.

Finally we turn to the case of FSR for the valence gluon propagating in the background nonabelian field. Here we only quote the result for the gluon Green's function in the background Feynman gauge 618 We have

$$
G_{\mu \nu}(x, y)=\left\langle x\left|\left(D_{\lambda}^{2} \delta_{\mu \nu}-2 i g F_{\mu \nu}\right)^{-1}\right| y\right\rangle
$$

Proceeding in the same way as for quarks, one obtains the FSR for the gluon Green's function

$$
G_{\mu \nu}(x, y)=\int_{0}^{\infty} d s(D z)_{x y} e^{-K_{0}} \Phi_{\mu \nu}(x, y)
$$

where we have defined

$$
\begin{aligned}
K_{0} & =\frac{1}{4} \int_{0}^{\infty}\left(\frac{d z_{\mu}}{d \tau}\right)^{2} d \tau \\
\Phi_{\mu \nu}(x, y) & =\left[P_{A} \exp \left(i g \int_{y}^{x} A_{\lambda} d z_{\lambda}\right) P_{F} \exp \left(2 g \int_{0}^{s} d \tau F_{\sigma \rho}(z(\tau))\right)\right]_{\mu \nu} .
\end{aligned}
$$

Now in the same way as is done above for scalars in Eq. (7), one may consider a Green's function, corresponding to the physical transition amplitude from a white state of $q_{1}, \bar{q}_{2}$ to another white state consisting of $q_{3}, \bar{q}_{4}$. It is given by

$$
G_{q \bar{q}}^{\Gamma}(x, y)=\left\langle G_{q}(x, y) \Gamma G_{\bar{q}}(x, y) \Gamma-G_{q}(x, x) \Gamma G_{\bar{q}}(y, y) \Gamma\right\rangle_{A},
$$

where $\Gamma$ describes the interaction between the $q, \bar{q}$ pair in the meson. The first term on the r.h.s. of Eq. (14) can be reduced to the same form as in Eq. (7) but with the Wilson loop containing ordered insertions of the operators $\sigma_{\mu \nu} F_{\mu \nu}$ (cf. Eq. 100). 


\section{Perturbation theory in the framework of FSR. Identities and partial summation.}

In this section we discuss in detail how the usual results of perturbation theory follow from FSR. It is useful to establish such a general connection between the perturbation series (Feynman diagram technique) and FSR. At the same time the FSR presents a unique possibility to sum up Feynman diagrams in a very simple way where the final result of the summation is written in an exponentiated way 16.18 This method will be discussed in the next section.

Consider the FSR for the quark Green's function. According to (9), the 2-nd order of perturbative expansion of Eq. (10) can be written as

$$
\begin{aligned}
G_{q}(x, y) & =\left(m_{q}-\hat{D}\right) \int_{0}^{\infty} d s \int_{0}^{\infty} d \tau_{1} \int_{0}^{\infty} d \tau_{2} e^{-K}(D z)_{x u} d^{4} u(D z)_{u v} d^{4} v(D z)_{v y} \\
& \times\left(i g A_{\mu}(u) \dot{u}_{\mu}+g \sigma_{\mu \nu} F_{\mu \nu}(u)\right)\left(i g A_{\nu}(v) \dot{v}_{\nu}+g \sigma_{\lambda \sigma} F_{\lambda \sigma}(v)\right)
\end{aligned}
$$

where we have used the identities

$$
\begin{gathered}
(D z)_{x y}=(D z)_{x u\left(\tau_{1}\right)} d^{4} u\left(\tau_{1}\right)(D z)_{u\left(\tau_{1}\right) v\left(\tau_{2}\right)} d^{4} v\left(\tau_{2}\right)(D z)_{v\left(\tau_{2}\right) y} \\
\int_{0}^{\infty} d s \int_{0}^{s} d \tau_{1} \int_{0}^{\tau_{1}} d \tau_{2} f\left(s, \tau_{1}, \tau_{2}\right)=\int_{0}^{\infty} d s \int_{0}^{\infty} d \tau_{1} \int_{0}^{\infty} d \tau_{2} f\left(s+\tau_{1}+\tau_{2}, \tau_{1}+\tau_{2}, \tau_{2}\right) .
\end{gathered}
$$

One can also expand only in the color magnetic moment interaction $(\sigma F)$. This is useful when the spin-dependent interaction can be treated perturbatively, as it is in most cases for mesons and baryons (exclusions are Goldstone bosons and nucleons, where spin interaction is very important and interconnected with chiral dynamics). In this case one obtains to the second order in $(\sigma F)$

$$
\begin{gathered}
G_{q}^{(2)}(x, y)=i\left(m_{q}-\hat{D}\right) \int_{0}^{\infty} d s \int_{0}^{\infty} d \tau_{1} \int_{0}^{\infty} d \tau_{2} e^{-m_{q}^{2}\left(s+\tau_{1}+\tau_{2}\right)-K_{0}-K_{1}-K_{2}} \\
(D z)_{x u} \Phi(x, u) g(\sigma F(u)) d^{4} u(D z)_{u v} \Phi(u, v) g(\sigma F(v)) d^{4} v(D z)_{v y} .
\end{gathered}
$$

In another way it can be written as

$$
\begin{gathered}
G_{q}^{(2)}(x, y)=i\left(m_{q}-\hat{D}\right)\left(m_{q}^{2}-D_{\mu}^{2}\right)_{x u}^{-1} d^{4} u g(\sigma F(u))\left(m_{q}^{2}-D_{\mu}^{2}\right)_{u v}^{-1} d^{4} v \\
\times g(\sigma F(v))\left(m_{q}^{2}-D_{\mu}^{2}\right)_{v y}^{-1} .
\end{gathered}
$$

Here $\left(m_{q}^{2}-D_{\mu}^{2}\right)^{-1}$ is the Green's function of a scalar quark in the external gluonic field $A_{\mu}$. This type of expansion is useful also for the study of small-x behavior of static potential, since the correlator $\langle\sigma F(u) \sigma F(v)\rangle$ plays an important role there. 
However, in establishing the general connection between perturbative expansion for Green's functions in FSR and expansions of exponential $\Phi_{\sigma}$ in Eq. (10), one encounters a technical difficulty since the coupling constant $g$ enters in three different ways in FSR:

1. in the factor $\left(m_{q}-\hat{D}\right)$ in front of the integral in Eq. (9)

2. in the parallel transporter (the first exponential in Eq. (10))

3 . in the exponential of $g(\sigma F)$.

Therefore it is useful to compare the two expansions in the operator form, the standard one

$$
\begin{aligned}
(m+\hat{D})^{-1}= & (m+\hat{\partial}-i g \hat{A})^{-1}=(m+\hat{\partial})^{-1}+(m+\hat{\partial})^{-1} i g \hat{A}(m+\hat{\partial})^{-1}+ \\
& +(m+\hat{\partial})^{-1} i g \hat{A}(m+\hat{\partial})^{-1} i g \hat{A}(m+\hat{\partial})^{-1}+\ldots
\end{aligned}
$$

and the FSR

$$
(m+\hat{D})^{-1}=(m-\hat{D})\left(m^{2}-\partial^{2}\right)^{-1} \sum_{n=o}^{\infty}\left(\delta\left(m^{2}-\partial^{2}\right)^{-1}\right)^{n},
$$

where we have introduced

$$
\delta=-i g(\hat{A} \hat{\partial}+\hat{\partial} \hat{A})-g^{2} \hat{A}^{2} \equiv \hat{D}^{2}-\partial^{2} .
$$

To see how the expansion (21) works, using $\hat{D}=\hat{\partial}-i g \hat{A}$ Eq. (21) becomes

$$
(m+\hat{D})^{-1}=\left[(m+\hat{\partial})^{-1}+i g \hat{A}\left(m^{2}-\partial^{2}\right)^{-1}\right] \sum_{n=0}^{\infty}\left[\delta\left(m^{2}-\partial^{2}\right)^{-1}\right]^{n}
$$

Separating out the first term we may rewrite this as

$(m+\hat{D})^{-1}=(m+\hat{\partial})^{-1}+(m+\hat{\partial})^{-1} i g \hat{A}(m-\hat{D})\left(m^{2}-\partial^{2}\right)^{-1} \sum_{n=0}^{\infty}\left[\delta\left(m^{2}-\partial^{2}\right)^{-1}\right]^{n}$

The last three factors in Eq. (23) are the same as occurring in Eq. (21). As a consequence the formal iteration of the resulting equation for the Greens' function reproduces the same series as in Eq. (20), showing the equivalence of the two expansions.

It is important to note that each term in the expansion in powers of $\delta$, after transforming the operator form of Eq. (21) into the integral form of FSR, becomes an expansion of the exponential $\Phi_{\sigma}$ in Eq. (10) in powers of $g$. The second order term of this expansion was written down before in Eq. (15). 
It is our purpose now to establish the connection between the expansion (21), (23) and the expansion of the exponential $\Phi_{\sigma}$ in Eq. (10) in the quark propagator (9). One can start with term linear in $\hat{A}$ and write (for the Abelian case see Appendix B of Ref. 19)

$$
G_{q}^{(1)}=i g \int G_{q}^{(0)}\left(x, z\left(\tau_{1}\right)\right) d^{4} z \frac{\xi_{\mu}(n)}{\varepsilon} \bar{A}_{\mu}\left(\tau_{1}\right) G_{q}^{(0)}\left(z\left(\tau_{1}\right), y\right)
$$

where the notation is clear from the general representation of $G_{q}$, given by Eq. (9)

$$
G_{q}(x, y)=\int_{0}^{\infty} d s e^{-s m_{q}^{2}} \prod_{n=1}^{N} \frac{d^{4} \xi(n)}{(4 \pi \varepsilon)^{2}} \exp \left[-\sum_{n=1}^{N} \frac{\xi^{2}(n)}{4 \varepsilon}\right] \Phi_{\sigma}(\bar{A}, \xi)
$$

with $\xi(n)=z(n)-z(n-1), \quad \bar{A}_{\mu}(n)=\frac{1}{2}\left[A_{\mu}(z(n))+A_{\mu}(z(n-1))\right]$ and

$$
\Phi_{\sigma}(\bar{A}, \xi)=P \exp \left\{i g \sum_{n=1}^{N} \bar{A}_{\mu}(n) \xi_{\mu}(n)+g \sum_{n=1}^{n} \sigma_{\mu \nu} F_{\mu \nu}(z(n)) \varepsilon\right\} .
$$

Representing $\xi(n)$ in Eq. (24) as $\frac{1}{2}\left(\xi_{\mu}(L)+\xi_{\mu}(R)\right)$, where $\xi_{\mu}(L)$ refers to the integral over $\xi_{\mu}$ in $G_{q}^{(0)}$ to the left of $\xi_{\mu}$ in Eq. (24) and $\xi_{\mu}(R)$ to the integral in $G_{q}^{(0)}$ standing to the right of $\xi_{\mu}$, we obtain

$$
\int \xi_{\mu}(n) \frac{d^{4} \xi(n)}{(4 \pi \varepsilon)^{2}} e^{i p \xi-\frac{\xi^{2}}{4 \varepsilon}}=-i \frac{\partial}{\partial p_{\mu}} e^{-i p^{2} \varepsilon}=2 i p_{\mu} \varepsilon e^{-p^{2} \varepsilon} .
$$

Thus Eq. (24) in momentum space becomes

$$
G_{q}^{(1)}=-g G_{q}^{(0)}(q)\left\langle q\left|p_{\mu} A_{\mu}+A_{\mu} p_{\mu}\right| q^{\prime}\right\rangle G_{q}^{(0)}\left(q^{\prime}\right)
$$

In a similar way the second order term from the coinciding arguments yields

$$
G_{q}^{(2)}(\text { coinc })=-g^{2} \int G_{q}^{(0)}(x, z) A_{\mu}^{2}(z) d^{4} z G_{q}^{(0)}(z, y)
$$

Finally, the first order expansion of the term $\sigma_{\mu \nu} F_{\mu \nu}$ in Eq. (10) yields the remaining missing component of the combination $\delta$, Eq. (22), which can be rewritten as

$$
\delta=-i g\left(A_{\mu} \partial_{\mu}+\partial_{\mu} A_{\mu}\right)-g^{2} A_{\mu}^{2}+g \sigma_{\mu \nu} F_{\mu \nu} .
$$

Hence the second term in the expansion (21)

$$
(m+\hat{D})^{-1}=(m-\hat{D})\left(m^{2}-\partial^{2}\right)^{-1}+(m-\hat{D})\left(m^{2}-\partial^{2}\right)^{-1} \delta\left(m^{2}-\partial^{2}\right)^{-1}+\ldots
$$


is exactly reproduced by the expansion of the FSR (9), where in the first exponential $\Phi_{\sigma}$ in Eq. (9) one keeps terms of the first and second order, $O\left(g A_{\mu}\right)$ and $O\left(\left(g A_{\mu}\right)^{2}\right)$, while in the second exponential one keeps only the first order term $O\left(g \sigma_{\mu \nu} F_{\mu \nu}\right)$. It is easy to see that this rule can be generalized to higher orders of the expansion in $\delta$ in Eq. (21) as well.

\section{Summing up leading perturbative contributions in FSR. Sudakov asymptotics.}

Consider now the n-point Green's function with external momenta $p_{i}$ at the i-th vertex

$$
G\left(p_{1}, \ldots p_{n}\right)=<J_{1}\left(p_{1}\right) \ldots J_{n}\left(p_{n}\right)>, J_{i}(x)=\psi(x)^{\dagger} \Gamma_{i} \psi(x)
$$

Insertion of Eq. (9) into Eq. (32) for the one-fermion loop yields

$$
\begin{aligned}
G\left(p_{1}, \ldots p_{n}\right) & =<\operatorname{tr} \prod_{i=1}^{n} \Gamma_{i}\left(m_{i}-\hat{D}_{i}\right) \int_{0}^{\infty} d s_{i} \\
& \times\left(D z^{(i)}\right)_{x^{(i)}, x^{(i-1)}} e^{-K_{i}} \Phi_{\sigma}^{(i)} e^{i p^{(i)} x^{(i)}} d x^{(i)}>_{A}
\end{aligned}
$$

We shall disregard in what follows the factors $\Gamma_{i}\left(m_{i}-\hat{D}_{i}\right)$ since we shall be interested only in the exponentiated contributions. Performing the $d x^{(i)}$ integrals, one obtains

$$
G \rightarrow \bar{G}_{n} \delta\left(\sum_{i=1}^{n} p_{i}\right)(2 \pi)^{4},
$$

where

$$
\bar{G}_{n}=\int \frac{d^{4} q}{(2 \pi)^{4}} \prod_{i=1}^{n} d s_{i} \prod_{k=1}^{N} \frac{d \xi^{(i)}(k)}{(4 \pi \varepsilon)^{2}} e^{i q^{(i)} \sum_{k} \xi^{(i)}(k)} e^{-K_{i}}<\Gamma_{i}\left(m_{i}-\hat{D}_{i}\right) W_{\sigma}>
$$

with

$$
<W_{\sigma}>=<\prod_{i=1}^{n} \Phi_{\sigma}^{(i)}>_{A},
$$

where $q^{(i)}$ is the momemtum of the fermion loop going from the i-th to the $(\mathrm{i}+1)$-th vertex. The integral $d^{4} q$ denotes the integral over one of $q^{(i)}$, all others being expressed through it and all $p_{i}$.

We note that $\left\langle W_{\sigma}\right\rangle$ is a gauge invariant quantity summing all the perturbative exchanges inside the fixed Wilson contour, defined by the set 
$\left\{\xi^{(i)}(k)\right\}$. In addition to the usual Wilson (charge) vertices, there are also magnetic moment vertices $\sigma F$, hence the notation $\left\langle W_{\sigma}\right\rangle$.

We concentrate now on the contribution of the $A_{\mu}$ field in (34), yielding the dominant contribution in the asymptotics (the reader is referred for the discussion of the $\sigma F$ term to the Appendix of Ref. 16 .

The crucial step for what follows is the use of the cluster expansion method which yields for $\left\langle W_{\sigma}\right\rangle \rightarrow\langle W\rangle$

$$
<W>\equiv \exp \left\{\sum_{r=1}^{\infty} \frac{(i g)^{r}}{r !} \sum_{k_{i}} \xi_{\mu_{1}}\left(k_{1}\right) \xi_{\mu_{2}}\left(k_{2}\right) \ldots \xi_{\mu_{r}}\left(k_{r}\right) \ll A_{\mu_{1}}\left(z_{k_{1}}\right) \ldots A_{\mu_{r}}\left(z_{k_{r}}\right) \gg\right\}
$$

Here double brackets denote cumulants 1 The lowest order contribution (in the exponent) can be expressed through the photon (gluon) propagator. In the Feynman gauge it is (the gauge is irrelevant since $\langle W\rangle$ is gauge invariant)

$$
<A_{\mu}(z) A_{\nu}\left(z^{\prime}\right)>=\frac{\delta_{\mu \nu} C_{2}(f) \hat{1}}{4 \pi^{2}\left(z-z^{\prime}\right)^{2}} .
$$

Here $C_{2}(f)$ is the quadratic Casimir operator for the fundamental representation, $\hat{1}$ is the unit color matrix. For QED one should replace $C_{2} \hat{1} \rightarrow 1$.

Eq. (35) represents the perturbative sum in the exponent, which by itself is an important advantage, since each term of perturbative expansion is already exponentiated. This property of exponentiation is well known for the Wilson loop, without using the powerful cluster expansion technique. In particular, the static $Q \bar{Q}$ potential exponentiates and it can be defined through the Wilson loop as follows

$$
V(R)=-\lim _{T \rightarrow \infty} \frac{1}{T} \ln W(R, T) .
$$

In our case of possibly light quarks with masses $m_{i}$ one should carry out an additional integration over quark trajectories (i.e. over $d \xi^{(i)}(k)$ ). In this section we consider the asymptotics of the amplitude (34) and therefore use the stationary point analysis for trajectories $\left\{\xi^{(i)}(k)\right\}$. Instead in the next section another method will be exploited, which was used for partial summation of perturbative diagrams in Refs. 9-11. In that section we shall also show how to modify this method to include also nonperturbative contributions.

In what follows we confine ourselves to the lowest contribution (36) in Eq. (35) and show that it yields the double logarithmic asymptotics. First of all one can persuade oneself that the approximation (36) yields in Eq. (34) all diagrams with exchanges of photon/gluon lines between fermion lines, all orderings of lines included. For QCD this means the following: all orderings, i.e. 
all intersection of gluon lines in space-time are included, except that the color ordering of operators $t^{a}$ is kept fixed. Since the commutator of any two generators $t^{a}$ is subleading at large $N_{c}$, it means that Eq. (36) sums up all exchanges including intersections of gluonic lines in the leading $N_{c}$ approximation

Our next point is the integration over $d \xi(k)$ in Eq. (34) which is Gaussian in the main term $K_{i}$, defining the measure of integration. Therefore we can carry it out by expanding the exponent in Eq. (34) around the stable fixed point $\bar{\xi}$, which is obtained by differentiating the exponent in (34) with respect to $\xi^{(i)}(k)$. One has

$$
\begin{gathered}
\bar{\xi}^{(i)}(k)=2 \varepsilon_{i}\left\{i q^{(i)}-\frac{g^{2} C_{2}(f)}{4 \pi^{2}} \sum_{j, k^{\prime}} \frac{\bar{\xi}^{(j)}\left(k^{\prime}\right)}{\left(\bar{z}^{(i)}(k)-\bar{z}^{j}\left(k^{\prime}\right)\right)^{2}}+\right. \\
\left.+\frac{2 g^{2} C_{2}(f)}{4 \pi^{2}} \sum_{j, k^{\prime}} \sum_{m \geq k} \frac{\left(\bar{\xi}^{(i)}(m) \bar{\xi}^{(j)}\left(n^{\prime}\right)\right)\left(\bar{z}^{(i)}(m)-\bar{z}^{(j)}\left(n^{\prime}\right)\right)}{\left(\bar{z}^{(i)}(m)-\bar{z}^{(j)}\left(n^{\prime}\right)\right)^{4}}\right\}+0\left(g^{4}\right)
\end{gathered}
$$

Here e.g. $\bar{z}^{(i)}(k)=\sum_{j=1}^{i} \sum_{\nu=1}^{k} \bar{\xi}^{(j)}(\nu)$, where we have chosen as the origin the coordinate $x^{(1)}$ of the first vertex, and all other coordinates are calculated using the connection $x^{(i)}-x^{(i-1)}=\sum_{k=1}^{N} \xi^{(i)}(k)$ with the cyclic condition $x^{(n+1)}=x^{(1)}$.

One can solve the system of equations (38) iteratively expanding in powers of $g^{2}$. The first two terms are given in (38), where one should replace $\bar{\xi}^{(i)}$ inside the curly brackets by $2 i \varepsilon_{i} q^{(i)}$. If one represents the exponential appearing in Eq. (34) after insertion of Eq. (35) as $\exp (-f(\xi, q))$, then one can write

$$
\begin{aligned}
f(\xi, q)= & \sum_{i, k} \frac{\left(\xi^{(i)}(k)\right)^{2}}{4 \varepsilon_{i}}-i \sum_{i, k} q^{(i)} \xi^{(i)}(k) \\
& -\frac{g^{2} C_{2}(f)}{8 \pi^{2}} \sum_{i, j, k k^{\prime}} \frac{\xi^{i}(k) \xi^{j}\left(k^{\prime}\right)}{\left(z^{i}(k)-z^{j}\left(k^{\prime}\right)\right)^{2}}+0\left(g^{4}\right) .
\end{aligned}
$$

The Gaussian integration in (34) finally yields

$$
\bar{G}_{n} \sim \int \frac{d^{4} q}{(2 \pi)^{4}} \prod_{i=1}^{n} d s_{i} e^{-f(\bar{\xi}, q)-\frac{1}{2} \operatorname{tr} \ln \varphi},
$$

where the matrix $\varphi$ is

$$
\varphi_{k n}^{i j}=\left.\frac{1}{2} \frac{\partial^{2}}{\partial \xi^{(i)}(k) \partial \xi^{(j)}(n)} f(\xi, q)\right|_{\xi=\bar{\xi}} .
$$


The most important for what follows is the term $f(\bar{\xi}, q)$ which can be written as (at this point we reestablish Minkowskian metric)

$$
f(\bar{\xi}, q)=\sum_{i=1}^{n} s_{i}\left(q^{(i)}\right)^{2}+\frac{g^{2} C_{2}(f)}{8 \pi^{2}} \sum_{i j} \int_{0}^{s_{i}} \int_{0}^{s_{j}} \frac{d \tau_{i} d \tau_{j}\left(q^{(i)} q^{(j)}\right)}{\left(\tau_{i} q^{(i)}-\tau_{j} q^{(j)}-\Delta_{i j}\right)^{2}},
$$

where we have defined $\tau_{i}=k \varepsilon_{i}$, and

$$
\Delta_{i j}=\sum_{k=i}^{j-1} s_{k} q^{(k)}, i<j .
$$

The integral in the last term on the r.h.s. of Eq. (42) can be written as $s_{i} s_{j}\left(q^{(i)} q^{(j)}\right) I_{i j}(s, q)$, where

$$
I_{i j}(s, q)=\int_{0}^{1} \int_{0}^{1} \frac{d \alpha d \beta}{\left(\alpha s_{i} q^{(i)}-\beta s_{j} q^{(j)}-\Delta_{i j}\right)^{2}} .
$$

The diagonal terms, $I_{i j}$, with $i=j$ do not contribute to the asymptotics and contain only selfenergy divergencies, which are of no interest to us in what follows. Therefore we shall consider only the nondiagonal terms with $i \neq j$.

Let us first study the term with $i=j-1$ ("the dressed vertex contribution") and $\Delta_{i, i+1}=s_{i} q^{(i)}$. Then Eq. (44) is reduced to the form which will be studied below

$$
\begin{gathered}
I_{i} \equiv I_{i, i+1}(s, q)=\int_{0}^{1} \int_{0}^{1} \frac{d \alpha d \beta}{\left(\alpha s_{i} q^{(i)}+\beta s_{j} q^{(j)}\right)^{2}} \\
=\int_{0}^{1} \int_{0}^{1} \frac{d \alpha d \beta}{\left(a^{2} \alpha^{2}+\beta^{2} b^{2}+2 \alpha \beta(a b)\right)}
\end{gathered}
$$

with $a=s_{i} q^{(i)}, b=s_{j} q^{(j)}, j=i+1$. As it stands the integral (45) diverges at small $\alpha, \beta$ (or at small $\tau_{i}, \tau_{j}$ in Eq. (42)). The origin of this divergence becomes physically clear, when one expresses the distance $z^{(i)}$ from the vertex position, (we go over to the Minkowskian space-time)

$$
z^{(i)}=2 q^{(i)} \tau_{i}, \quad z^{(j)}=2 q^{(j)} \tau_{j} .
$$

The quasiclassical motion (46) cannot be true for small $\tau_{i}$, when quantum fluctuations wash out the straight-line trajectories. The lower limit $\tau_{\min }$ can be obtained from the quantum uncertainty principle

$$
\Delta z \Delta q \sim\left(z^{(i)}-z^{(j)}\right)\left(q^{(i)}-q^{(j)}\right) \sim 1
$$


Furthermore, we shall be interested in the kinematical region, where

$$
\left|q^{(i)} q^{(j)}\right| \gg\left(q^{(i)}\right)^{2},\left(q^{(j)}\right)^{2} .
$$

The value of $\tau_{\min }$ then is found from (47) to be

$$
\tau_{\min } \sim \frac{1}{2\left|q^{(i)} q^{(j)}\right|} .
$$

Using Eq. (49) one can easily calculate the integral (45), since the term $2 \alpha \beta(a b)$ in the denominator of the integrand in Eq. 45) always dominates. The result is

$$
I_{i}=\frac{1}{2 s_{i} s_{i+1}\left(q^{(i)} q^{(i+1)}\right)} \ln \left(2\left(q^{(i)} q^{(i+1)}\right) s_{i}\right) \ln \left(2\left(q^{(i)} q^{(i+1)}\right) s_{i+1}\right) .
$$

The integration of the general term $I_{i j}$ with $j \neq i-1, i+1$ can be done using the expressions for the Spence functions. However in the general case the lower limit $\tau_{\min }$ is inessential and the double logarithmic situation does not occur unless there is a large ratio, $\left|\frac{\left(q_{i} q_{k}\right)}{\left(q_{l} q_{m}\right)}\right| \gg 1$.

We start with the open triangle, corresponding to the Sudakov vertex function asymptotics, i.e. when the fermion loop is not closed. We have

$$
\bar{G}_{3}=(-i \hat{q}+m)^{-1} \Gamma\left(q, q^{\prime}\right)\left(-i \hat{q}^{\prime}+m\right)^{-1} .
$$

In this case there is no integration over $d^{4} q$ in Eq. (34) and only one integral $I_{12}$ is present in Eqs. (44) and (50) (we disregard as before the selfinteracting pieces $I_{i i}$, which do not contribute to the asymptotics).

Inserting Eq. (50) into Eq. (42) and integration over $d s_{1} d s_{2}$ in Eq. (40) yields the leading contribution, where in Eq. (50) the arguments $s_{i}$ are taken to be $s_{i}=\frac{1}{q_{i}^{2}}$. For the case of $\operatorname{QED}\left(C_{2} \equiv 1\right)$ we find

$$
\Gamma\left(q, q^{\prime}\right) \sim \exp \left(-\frac{\alpha}{2 \pi} \ln \frac{2\left|q q^{\prime}\right|}{q^{2}} \ln \frac{2\left|q q^{\prime}\right|}{\left(q^{\prime}\right)^{2}}\right),
$$

which coincides with the known Sudakov asymptotics.

We turn now to the case of QCD, where the basic triangle diagram is closed due to color gauge invariance and try to find out whether the kinematical region (48) plays an important role in the integral over $d^{4} q$ in Eq. (40).

In the general case, when all $q_{i}$ are unconstrained and expressed through three external momenta $p_{1}, p_{2}, p_{3}$ and one integration variable, the region (48), yielding double logarithmic asymptotics (DLA) (50), is suppressed due to large values of $f(\bar{\xi}, q)$ in the exponent. As a result the integral over $d q$ does not lead to the DLA form for $\bar{G}_{3}$. 
The situation changes however, if one considers instead of $\bar{G}_{3}$ the form factor, i.e. when the pole terms are factored out from the vertices 2 and 3 and the vertex functions appear there. To simplify matter, one can consider for the form factor the same representation (40). Under the integral one has the vertex functions $\psi_{i}\left(k_{i}\right), i=1,3$, where

$$
\begin{aligned}
& k_{1}=q^{(1)}+q^{(3)}-p^{(1)} \frac{\left(q^{(1)}+q^{(3)}\right) p^{(1)}}{\left(p^{(1)}\right)^{2}}, \\
& k_{3}=q^{(2)}+q^{(3)}-p^{(3)} \frac{\left(q^{(2)}+q^{(3)}\right) p^{(3)}}{\left(p^{(3)}\right)^{2}} .
\end{aligned}
$$

The definition (53) yields in the c.m. system of particle 1 or 3 the familiar relative momentum of two emitted fermions. The presence of $\psi_{i}$ imposes a restriction on the momenta $q_{i}$, namely

$$
k_{1}^{2}, k_{3}^{2} \lesssim \kappa^{2}
$$

where $\kappa^{2}$ is some hadronic scale.

Let us define in the Breit system the momenta

$$
p^{(1)}, p^{(2)}, p^{(3)}=\left(p_{0},-\frac{\vec{Q}}{2}\right),(0, \vec{Q}),\left(p_{0}, \frac{\vec{Q}}{2}\right),
$$

where $\vec{Q}^{2} \gg \kappa^{2}$ and $p_{0}^{2}=M^{2}+\frac{\vec{Q}^{2}}{4}$. One can then easily see, that Eq. (54) constrains the region of integration over $d q \equiv d^{4} q_{1}$ to the region $|\vec{q}| \sim \kappa, \mid q_{0}-$ $p_{0} \mid \sim \kappa$, and the conditions (48) are satisfied. Hence in this case one recovers the Sudakov asymptotics (52), where

$$
\left|q q^{\prime}\right| \rightarrow\left|q^{(1)} q^{(2)}\right| \approx \frac{\vec{Q}^{2}}{4}, q^{2} \sim q^{\prime 2} \sim \kappa^{2}, \quad \alpha \rightarrow \alpha_{s} C_{2} .
$$

\section{Explicit path integration in FSR. Connection to the Bern-Kosower- Strassler method.}

The path integral (9) can be performed in a direct way. After the expansion of Eq. (10) in powers of $A_{\mu}$ and $F_{\mu \nu}$ and exploiting Fourier transform for the latter one obtains Gaussian integrals which can be easily done.

This procedure is similar to the method introduced in Refs. 9-11 for oneloop diagrams and the effective action in QED and QCD. To this end we extend the method of Refs. 9-11 to the case of meson and glueball Green's functions. We will show, that the method used in Refs. 9-11 can be simplified and generalized. 
Let us start with three typical expressions in the order of increasing complexity. We may consider the one-loop effective action

$$
\Gamma\{A\}=\int_{0}^{\infty} \frac{d s}{s} \xi(s) e^{-m^{2} s-\frac{1}{4} \int_{0}^{s} \dot{z}_{\mu}^{2} d \tau}(D z)_{x x} t r W\left(A_{\mu}\right),
$$

where $\xi(s)$ is a regularization. Other expressions, which may be studied, are the heavy-light quark-antiquark Green's function

$$
G_{H L}(Q)=\int_{0}^{\infty} d s e^{-m^{2} s-\frac{1}{4} \sum_{n=1}^{N} \frac{\xi^{2}(n)}{\varepsilon}-i Q \sum_{n=1}^{N} \xi(n)} \prod_{n=1}^{N} \frac{d^{4} \xi(n)}{(4 \pi \varepsilon)^{2}}\left\langle W\left(A_{\mu}\right)\right\rangle .
$$

or the Green's function for the arbitrary mass quark-antiquark system

$$
\begin{aligned}
G(Q)= & \int_{0}^{\infty} d s_{1} \int_{0}^{\infty} d s_{2} \frac{d^{4} p}{(2 \pi)^{4}} e^{-i p_{\mu} \sum_{n_{1}=1}^{N_{1}} \xi_{\mu}^{(1)}\left(n_{1}\right)-i\left(Q_{\mu}-p_{\mu}\right) \sum_{n_{2}=1}^{N_{2}} \xi_{\mu}^{(2)}\left(n_{2}\right)} \\
& \times e^{-m_{1}^{2} s_{1}-m_{2}^{2} s_{2}} \prod_{n_{1}=1}^{N_{1}} \frac{d^{4} \xi^{(1)}\left(n_{1}\right)}{\left(4 \pi \varepsilon_{1}\right)^{2}} \prod_{n_{2}=1}^{N_{2}} \frac{d^{4} \xi^{(2)}\left(n_{2}\right)}{\left(4 \pi \varepsilon_{2}\right)^{2}}\left\langle\operatorname{tr} W\left(A_{\mu}\right)\right\rangle .
\end{aligned}
$$

Here we have introduced $N_{1} \varepsilon_{1}=s_{1}, N_{2} \varepsilon_{2}=s_{2}$.

Let us consider the expression (57), since Eq. (56) can be managed easily in the same way. To proceed one expands in (Eq. 57) $\left\langle W\left(A_{\mu}\right)\right\rangle$ in powers of $A_{\mu}$ and uses the Fourier transform $\tilde{A}_{\mu}(k)$ for the latter. To the second order in $g$ one obtains

$$
\begin{gathered}
G_{H L}(Q)-G_{H L}^{(0)}(Q)=-g^{2} \int_{0}^{\infty} d s e^{-m^{2} s-i Q \sum \xi(n)-\frac{1}{4} \sum \frac{\xi^{2}(n)}{\varepsilon}} \\
\times \prod_{n=1}^{N} \frac{d \xi(n)}{(4 \pi \varepsilon)^{2}} \int_{0}^{s} \dot{z}_{\mu}\left(t_{1}\right) d t_{1} \int_{0}^{t_{1}} \dot{z}_{\nu}\left(t_{2}\right) d t_{2}\left\langle\tilde{A}_{\mu}\left(k_{1}\right) \tilde{A}_{\nu}\left(k_{2}\right)\right\rangle \\
\times \frac{d^{4} k^{(1)} d^{4} k^{(2)}}{(2 \pi)^{8}} e^{i k_{\mu}^{(1)} z_{\mu}\left(t_{1}\right)+i k_{\mu}^{(2)} z_{\mu}\left(t_{2}\right)} .
\end{gathered}
$$

Defining similarly to Ref. 11

$$
\sum_{i=1}^{2} i k^{(i)} z\left(t_{i}\right)=i \int_{0}^{s} J_{\mu}(\tau) z_{\mu}(\tau) d \tau, \quad J_{\mu}(\tau)=k_{\mu}^{(1)} \delta\left(\tau-t_{1}\right)+k_{\mu}^{(2)} \delta\left(\tau-t_{2}\right)
$$


one arrives at the integral

$I(Q, J) \equiv \prod_{i=1}^{N} \frac{d^{4} \xi(i)}{(4 \pi \varepsilon)^{2}} \exp \left\{-i Q \sum_{n} \xi(n)-\frac{1}{4 \varepsilon} \sum_{n} \xi^{2}(n)+i \varepsilon \sum_{k=1}^{N} \xi_{\mu}(k) \sum_{n=k}^{N} J_{\mu}(n)\right\}$,

where we have used that $z_{\mu}(t)=\sum_{k=1}^{n} \xi_{\mu}(k)$ and interchanged the order of summation in $\int J_{\mu}(\tau) z_{\mu}(\tau) d \tau=\sum J_{\mu}(n) z_{\mu}(n) \varepsilon$.

The Gaussian integration in Eq. (61) can be performed trivially and one obtains

$$
\begin{aligned}
& I(Q, J)=\exp \left\{-\varepsilon^{3} \sum_{k=1}^{N} \sum_{n=k}^{N} \sum_{n^{\prime}=k}^{N} J_{\mu}(n) J_{\mu}\left(n^{\prime}\right)-Q^{2} s+\varepsilon^{2} 2 Q_{\mu} \sum_{k=1}^{N} \sum_{n=k}^{N} J_{\mu}(n)\right\} \\
& \quad=\exp \left\{-\int_{0}^{s} d \tau\left(\int_{\tau}^{s} J_{\mu}\left(\tau^{\prime}\right) d \tau^{\prime}\right)^{2}-Q^{2} s+2 Q_{\mu} \int_{0}^{s} d \tau \int_{\tau}^{s} J_{\mu}\left(\tau^{\prime}\right) d \tau^{\prime}\right\} .
\end{aligned}
$$

Substituting $(60)$ into $(62)$ one finally gets

$$
I(Q, J)=\exp \left\{-Q^{2} s+2 Q_{\mu}\left(k_{\mu}^{(1)} t_{1}+k_{\mu}^{(2)} t_{2}\right)-\sum_{i, j=1,2} k_{\mu}^{(i)} k_{\mu}^{(j)} g_{B}\left(t_{i}, t_{j}\right)\right\}
$$

where

$$
g_{B}\left(t_{i}, t_{j}\right)=\operatorname{Min}\left(t_{i}, t_{j}\right) .
$$

In the derivation of Eq. (63) one could apply the string vertex technique of Refs. 9-11. A corresponding method for the Dirac propagator was used in Ref. 20.

So far we have neglected the factors $\dot{z}_{\mu}\left(t_{1}\right) \dot{z}_{\nu}\left(t_{2}\right)$ in 59 . To take them into account one could exploit the trick suggested in Ref. 11. Instead we shall use below another approach, based on the following relation ( we denote $I_{\mu \nu}(Q, J)$ the integral 61 with $\dot{z}_{\mu} \dot{z}_{\nu}$ taken into account). Noting that $\dot{z}_{\mu}(t)=\frac{\xi_{\mu}(k)}{\varepsilon}$ we obtain

$$
\begin{aligned}
I_{\mu \nu}(Q, J) & =\prod_{n=1}^{N} \frac{d^{4} \xi(n)}{(4 \pi \varepsilon)^{2}} \frac{\xi_{\mu}\left(k_{1}\right)}{\varepsilon} \frac{\xi_{\nu}\left(k_{2}\right)}{\varepsilon} \\
& \times \exp \left[\sum_{k=1}^{N}\left(i \varepsilon \xi_{\mu}(k) j_{\mu}(k)-i Q \xi(k)-\frac{\xi^{2}(k)}{4 \varepsilon}\right)\right],
\end{aligned}
$$

where $j_{\mu}(k) \equiv \sum_{n=k}^{N} J_{\mu}(n)$. Hence one can make a simple connection

$$
I_{\mu \nu}(Q, J)=\left(\frac{1}{i \varepsilon^{2}} \frac{\partial}{\partial j_{\mu}\left(k_{1}\right)}\right)\left(\frac{1}{i \varepsilon^{2}} \frac{\partial}{\partial j_{\nu}\left(k_{2}\right)}\right) I(Q, J) .
$$


Computing (66) one should take into account that $t_{2} \leq t_{1}$. In doing so one obtains

$$
\dot{z}_{\mu}^{(1)} \rightarrow-2 i\left(Q_{\mu}-k_{\mu}^{(1)}\right) ; \quad \dot{z}_{\mu}^{(2)} \rightarrow-2 i\left(Q_{\mu}-k_{\mu}^{(1)}-k_{\mu}^{(2)}\right) .
$$

The $H L$ Green's function to the second order $O\left(g^{2}\right)$ has the form

$$
\begin{gathered}
G_{H L}(Q)-G_{H L}^{(0)}(Q)=4 g^{2} \int_{0}^{\infty} d s e^{-\left(m^{2}+Q^{2}\right) s} \frac{d^{4} k^{(1)} d^{4} k^{(2)}}{(2 \pi)^{8}}\left\langle\tilde{A}_{\mu}\left(k^{(1)}\right) \tilde{A}_{\nu}\left(k^{(2)}\right)\right\rangle \\
\times \int_{0}^{s} d t_{1} \int_{0}^{t_{1}} d t_{2}\left(Q_{\mu}-k_{\mu}^{(1)}\right)\left(Q_{\nu}-k_{\nu}^{(2)}-k_{\nu}^{(1)}\right) e^{2 Q_{\mu}\left(k_{\mu}^{(1)} t_{1}+k_{\mu}^{(2)} t_{2}\right)} \\
\times \exp \left[-\left(k^{(1)}\right)^{2} t_{1}-\left(k^{(2)}\right)^{2} t_{2}-2 k_{\mu}^{(1)} k_{\mu}^{(2)} t_{2}\right] .
\end{gathered}
$$

In some cases another form is convenient with integrals over $d t_{1} d t_{2}$ taken explicitly,

$$
\begin{gathered}
G_{H L}(Q)-G_{H L}^{(0)}(Q)=4 g^{2} \int \frac{d^{4} k^{(1)} d^{4} k^{(2)}}{(2 \pi)^{8}} \frac{\left\langle\tilde{A}_{\mu}\left(k^{(1)}\right) \tilde{A}_{\nu}\left(k^{(2)}\right)\right\rangle}{2 Q k^{(2)}-\left(k^{(2)}\right)^{2}-2 k^{(1)} k^{(2)}} \\
\times\left(Q_{\mu}-k_{\mu}^{(1)}\right)\left(Q_{\nu}-k_{\nu}^{(2)}-k_{\nu}^{(1)}\right)\left\{\frac { 1 } { 2 Q ( k ^ { ( 1 ) } + k ^ { ( 2 ) } ) - ( k ^ { ( 1 ) } + k ^ { ( 2 ) } ) ^ { 2 } } \left[-\frac{1}{m^{2}+Q^{2}}\right.\right. \\
\left.\left.+\frac{1}{m^{2}+\left(Q-k^{(1)}-k^{(2)}\right)^{2}}\right]-\frac{1}{2 Q k^{(1)}-\left(k^{(1)}\right)^{2}}\left[-\frac{1}{m^{2}+Q^{2}}+\frac{1}{m^{2}+\left(Q-k^{(1)}\right)^{2}}\right]\right\} .
\end{gathered}
$$

Eqs. (68) and (69) contain the general result for the contribution of the second order correlator to $G_{H L}(Q)$. One can further specify the correlator $\left\langle\tilde{A}_{\mu}\left(k^{(1)}\right) \tilde{A}_{\nu}\left(k^{(2)}\right)\right\rangle$. For example, for the perturbative propagator in the Feynman gauge one has

$$
\left\langle\tilde{A}_{\mu}\left(k^{(1)}\right) \tilde{A}_{\nu}\left(k^{(2)}\right)\right\rangle=\frac{4 \pi \delta_{\mu \nu}}{\left(k^{(1)}-k^{(2)}\right)^{2}},
$$

while for the nonperturbative correlator in the Balitsky gauge one obtains, using results from 21

$$
\begin{gathered}
\left\langle\tilde{A}_{\mu}(k) \tilde{A}_{\nu}\left(k^{\prime}\right)\right\rangle=\frac{\sigma e^{-\left(k_{4}-k_{4}^{\prime}\right)^{2} T_{g}^{2}}}{\sqrt{\pi} T_{g}} \int_{0}^{1} d t \int_{0}^{1} d t^{\prime} \alpha_{\mu}(t) \alpha_{\nu}\left(t^{\prime}\right) \\
\times\left(\delta_{\mu \nu} \delta_{i k}-\delta_{i \nu} \delta_{k \mu}\right)\left(i \frac{\partial}{\partial k_{i}} i \frac{\partial}{\partial k_{k}^{\prime}}\right) \exp \left\{-\left[\left(\alpha+\tilde{t}^{\prime 2}\right) \mathbf{k}^{2}+\left(\alpha+\tilde{t}^{2}\right) \mathbf{k}^{\prime 2}+\right.\right.
\end{gathered}
$$


18

$$
\left.\left.+2 \tilde{t} \tilde{t}^{\prime}\left(\mathbf{k} \cdot \mathbf{k}^{\prime}\right)\right]\left[4 \alpha\left(\alpha+\tilde{t}^{2}+\tilde{t}^{\prime 2}\right)\right]^{-1}\right\} \cdot\left[\alpha\left(\alpha+\tilde{t}^{2}+\tilde{t}^{\prime 2}\right)\right]^{-3 / 2} \text {. }
$$

Here we have introduced above the regularizing factor $\exp \left(-\alpha\left(\mathbf{z}^{2}+\mathbf{w}^{2}\right)\right)$ and used the notation

$$
\tilde{t}=\frac{t}{2 T_{g}}, \tilde{t}^{\prime}=\frac{t^{\prime}}{2 T_{g}} ; \quad \alpha_{\mu}(t): \alpha_{4}(t)=1, \alpha_{i}(t)=t, i=1,2,3 .
$$

It is assumed that the limit $\alpha \rightarrow 0$ is taken at the end of the calculations.

Inserting Eq. (71) into Eqs. (68) and (69) one obtains the lowest order nonperturbative contribution to $G_{H L}$, which can be studied for all values of $Q$. In particular, one can expand the result in powers of $1 / Q^{2}$ to compare with the standard OPE result, where the expansion starts with $\frac{\operatorname{tr}\left(F_{\mu \nu}(0)\right)^{2}}{Q 4}$. The kernel (71) is nonlocal and generates a series in powers of $\left(Q T_{g}\right)^{2}$. See Ref. 22 for further details.

The equations (59-69) obtained above are valid in the second order of expansion of $\left\langle\operatorname{tr} W\left(A_{\mu}\right)\right\rangle$ in $g A_{\mu}$. The method used above can easily be generalized to higher orders. Indeed, writing

$$
\begin{gathered}
\left\langle\operatorname{tr} W\left(A_{\mu}\right)\right\rangle=\sum_{n=0}^{\infty}(i g)^{n}\left\langle\operatorname { t r } \left\{\int_{0}^{s} A_{\mu_{1}}\left(k^{(1)}\right) \dot{z}_{\mu_{1}}\left(t_{1}\right) d t_{1} \int_{0}^{t_{1}} A_{\mu_{2}}\left(k^{(2)}\right) \dot{z}_{\mu_{2}}\left(t_{2}\right) d t_{2}\right.\right. \\
\left.\left.\ldots \int_{0}^{t_{n-1}} A_{\mu_{n}}\left(k^{(n)}\right) \dot{z}_{\mu_{n}}\left(t_{n}\right) d t_{n} e^{i \sum_{m=1}^{n} k^{(m)} z\left(t_{m}\right)} \prod_{i=1}^{n} \frac{d^{4} k^{(i)}}{(2 \pi)^{4}}\right\}\right\rangle,
\end{gathered}
$$

one can define similarly to Eq. (60) for the $n$-th term in Eq. 72

$$
J_{\mu}^{(n)}(\tau)=\sum_{i=1}^{n} k_{\mu}^{(i)} \delta\left(\tau-t_{i}\right)
$$

Doing the integrals over $\prod_{k=1}^{N} d \xi(k)$ as before (cf. Eq. (61)) one obtains the following result, generalizing Eq. 62,

$I^{(n)}(Q, J)=\exp \left\{-\int_{0}^{s} d \tau\left(\int_{\tau}^{s} j_{\mu}\left(\tau^{\prime}\right) d \tau^{\prime}\right)^{2}-Q^{2} s+2 Q_{\mu} \int_{0}^{s} d \tau \int_{\tau}^{s} J_{\mu}\left(\tau^{\prime}\right) d \tau^{\prime}\right\}$.

Inclusion of the terms $\dot{z}_{\mu_{1}}\left(t_{1}\right) \ldots \dot{z}_{\mu_{n}}\left(t_{n}\right)$ can be done in the same way as in Eq. (66) and the generalization of $I^{(n)}(Q, J)$ to $I_{\mu_{1} \ldots \mu_{n}}^{(n)}(Q, J)$ is straightforward. In this way one obtains the general form of the expansion of Green's functions (56) -58 ) to all orders in $g$. In contrast to the results of Refs. 9-11 one keeps not only perturbative but also nonperturbative contributions. 
Although we have used here the FSR method to study the case of scalar quarks, it can also also be applied for quarks with spin starting from Eq. (18). We have shown in the present paper how to use the FSR to treat perturbative QCD, establishing in particular the correspondence between the standard and FSR perturbation expansion.

1. M.S. Marinov, Phys. Rept. 60C 1 (1980).

2. R.P. Feynman, Phys. Rev. 80440 (1950); ibid 84108 (1951).

3. V.A. Fock, Izvestya Akad. Nauk USSR, OMEN, 1937, p.557; J. Schwinger, Phys. Rev. 82664 (1951).

4. G.A. Milkhin and E.S. Fradkin, ZhETF 451926 (1963); E.S. Fradkin, Trudy FIAN, 297 (1965).

5. M.B. Halpern, A. Jevicki and P. Senjovic, Phys. Rev. D16 2474 (1977); K. Bardakci and S. Samuel, Phys.Rev. D18 2849 (1978); R. Brandt et al, Phys. Rev. D19 1153 (1979); S. Samuel, Nucl. Phys. B149 517 (1979); J. Ishida and A. Hosoya, Progr. Theor. Phys. 62544 (1979).

6. Yu.A. Simonov, Nucl. Phys. B307 512 (1988).

7. A.I. Karanikas and C.N. Ktorides, Phys. Lett. B275 403 (1992); Phys. Rev. D52 58883 (1995).

8. A.I. Karanikas, C.N. Ktorides and N.G. Stefanis, Phys. Rev. D52 5898 (1995).

9. Z. Bern and D.A. Kosower, Phys. Rev. Lett. 661669 (1991); Nucl. Phys. B379 451 (1992).

10. Z. Bern and D.C. Dunbar, Nucl. Phys. B379 562 (1992).

11. M.J. Strassler, Nucl. Phys. B385 145 (1992).

12. Yu.A. Simonov, JETP Lett 54249 (1991); ibid. 55 (1992) 627; Phys. At. Nucl. 58309 (1995).

13. Yu.A. Simonov, In "Varenna 1995, Selected topics in nonperturbative QCD", p.319.

14. H.G. Dosch, H.-J. Pirner and Yu.A. Simonov, Phys. Lett. B349 335 (1995); E.L. Gubankova and Yu.A. Simonov, Phys. Lett. 36093 (1995); N.O. Agasyan, in preparation.

15. N.G. van Kampen, Phys. Rept. C24 171 (1976).

16. Yu.A. Simonov, Phys. Lett. 464265 (1999).

17. B.S. De Witt, Phys. Rev. 16211951239 (1967);

J. Honerkamp, Nucl. Phys. B48 269 (1972);

G.'t Hooft, Nucl. Phys. B62 444 (1973); Lectures at Karpacz, in : Acta Univ. Wratislaviensis 368345 (1976);

L.F. Abbot, Nucl. Phys. B185 189 (1981).

18. Yu.A. Simonov, Phys. At Nucl. 58107 (1995); JETP Lett. 75525 (1993); 
Yu.A. Simonov, in: Lecture Notes in Physics v.479, p. 139; ed. H. Latal and W. Schwinger, Springer, 1996.

19. Yu.A. Simonov and J.A. Tjon, Ann. Phys. 2281 (1993).

20. M. Henneaux and C. Teitelboim, Ann. Phys. 143127 (1982);

V.Ya. Fainberg, A.Va. Marshakov, Nucl.Phys. B306 659 (1988).

21. Yu.A. Simonov, Phys. At. Nucl. 602069 (1997), hep-ph/9704301;

Yu.A. Simonov and J.A. Tjon, Phys. Rev. D62 014501 (2000).

22. V.I. Shevchenko and Yu.A. Simonov, hep-ph/0109051. 